\title{
Genèse et diversité des villes-usines européennes (XVIIIe-XXe siècles), une approche patrimoniale
}

Development and diversity of European Factory- towns $\left(18^{\text {th }}-19^{\text {th }}\right)$, a heritage approach

\section{Gracia Dorel-Ferré}

\section{OpenEdition}

Journals

Édition électronique

URL : https://journals.openedition.org/rge/9258

DOI : $10.4000 /$ rge.9258

ISSN : 2108-6478

Éditeur

Association des géographes de l'Est

\section{Référence électronique}

Gracia Dorel-Ferré, «Genèse et diversité des villes-usines européennes (XVIIIe-XXe siècles), une approche patrimoniale », Revue Géographique de l'Est [En ligne], vol. 58/3-4 | 2018, mis en ligne le 13 juillet 2020, consulté le 15 février 2023. URL : http://journals.openedition.org/rge/9258 ; DOI : https:// doi.org/10.4000/rge.9258

Ce document a été généré automatiquement le 8 octobre 2021.

Tous droits réservés 


\title{
Genèse et diversité des villes-usines européennes (XVIIIe-XXe siècles), une approche patrimoniale
}

Development and diversity of European Factory- towns $\left(18^{\text {th }}-19^{\text {th }}\right)$, a heritage approach

\author{
Gracia Dorel-Ferré
}

1 Depuis les travaux de Roger-Henri Guerrand sur le logement social (1967, 1989 et 1992), le sujet, étendu aux villages ouvriers et aux villes-usines de l'espace européen, a été largement exploré. On a redécouvert, en 1998, l'ouvrage pionnier qu'Henry Roberts avait publié en 1850 et augmenté en 1867 ; une bibliographie abondante s'est intéressée à des monographies comme à des études régionales telles que celles de Le Maner (1995) et Frouard (2008) pour le Bassin minier du Nord Pas de Calais ; Commaille pour le Grand Est (1999), Poull (1982) pour les vallées vosgiennes ou encore Jonas (2002) pour l'Europe centrale. Cependant on a inclus plus tardivement la dimension patrimoniale. Louis Bergeron comblera ce vide auprès d'ICOMOS (2001) et depuis, des synthèses, présentées dans le cadre des colloques organisés par $\mathrm{TICCIH}^{1}$, l'association internationale de patrimoine industriel, ont abouti à une publication globale (Dorel-Ferré, 2016).

2 Le village ouvrier, la ville-usine, sont des constructions d'initiative patronale, que le patron soit un individu, un groupe (association, syndicat), ou encore l'État. Leur diversité physionomique est remarquable, qu'ils soient édifiés à proximité d'une ressource (mines de charbon ou mines métalliques, carrières) ou d'une source d'énergie (eau, surtout). Leur finalité est d'accueillir une main d'œuvre qu'il faut aller chercher toujours plus loin, pour assurer une production de plus en plus importante. Avec l'évolution des conditions de vie dans un contexte d'industrialisation, l'habitat et sa disposition dans l'espace ont traduit non seulement des conditions locales concrètes, mais aussi des visions particulières de la société. Ainsi est-on passé, sans que cette évolution soit uniforme et progressive, de l'habitat en rang et de la caserne au village ouvrier et à la cité-jardin. Des expériences ont été réalisées qui n'ont pas eu de postérité, alors que d'autres formules ont connu un grand développement, comme c'est 
le cas de la cité Dollfus-Mieg, souvent copiée (Hau et Stroskopf, 2005). Au cours du XXe siècle, l'irruption de la voiture individuelle a également influé sur le mode de vie et sur l'organisation de l'espace, soit en accompagnant la formation de la cité-jardin soit en contribuant à déprécier le village industriel estimé trop proche du lieu de travail.

3 La physionomie de ces unités physionomiques et fonctionnelles peut varier suivant les matériaux de construction, les agencements du bâti et de la voierie, la localisation et la diversité des services, etc. On les identifie cependant facilement par leurs caractéristiques communes: les constructions de grande taille affectées aux acteurs économiques, religieux ou administratifs qui y exercent leur pouvoir ; l'homogénéité de l'habitat des ouvriers et employés, où la hiérarchisation des catégories socioprofessionnelles peut aller jusqu'à une réelle ségrégation (Söderström, 1997).

4 L'expérience anglaise a fasciné, du fait de sa précocité, dès le XVIIIe siècle, de sa cohérence, fondée sur l'emploi systématique de l'hydraulique et de la machine à vapeur. L'exportation des modèles d'usines, d'habitat ouvrier et de machines a été facilitée par les expositions universelles, véritables programmes publicitaires grandeur nature. Cependant, la diffusion de l'industrialisation dans le continent européen, suivant une chronologie qui n'a jamais été uniforme, a introduit des variantes dans le modèle. Pour certains, en particulier les historiens de l'Italie du Nord, cette industrialisation n'a fait que renforcer des caractères déjà en place depuis le XVIe siècle au moins (Fontana, 1997). Le débat, qui reste toujours ouvert, a également mis en lumière le rôle fondateur, au XVIIIe siècle, des monarchies bourboniennes d'Italie, de France et d'Espagne (Dorel-Ferré, 2006) dans la conception et la création des villages ouvriers et des villes-usines. C'est également au XVIIIe siècle que la Russie impériale, s'industrialise par la décision de Pierre le Grand et que se multiplient les villes-usines ouraliennes (Portal, 1950 ; Lotareva, 2011).

5 Peut-on confondre, dans une même acception, villages ouvriers et villes-usines ? Si les géographes ont déjà discuté le sujet (Edelblutte, 2010), les historiens, de leur côté (Garner, 1992), ont tenté de clarifier un concept somme tout assez versatile ${ }^{2}$. Certains auteurs (Jonas, 1985), ont introduit l'expression de «ville industrielle » distincte de la « cité industrielle » dont parle Lucie Morisset pour le Canada (Morisset, 1998).

6 L'historien John S. Garner, qui a signé l'un des ouvrages de référence sur le sujet, souligne combien les expressions anglo-saxonnes sont restrictives. Il rappelle que le terme de "company town" date de la fin du XIXe siècle et caractérise, tout d'abord, les implantations minières et métallurgiques des Appalaches fondées par des groupes d'investisseurs privés. Mais peut-il s'appliquer à une entreprise d'Etat, ou encore à une manufacture, privée ou privilégiée d'Ancien Régime ? Ce serait inapproprié, ou, à tout le moins, anachronique. Pourtant, c'est le terme employé par Garner, un peu par défaut, et qui est largement diffusé dans le domaine anglo-saxon (Fortier, 1996, Morisset, 1998). L'expression de cité ouvrière paraît mieux convenir, dit Garner, mais elle est proprement intraduisible dans les autres langues, où elle prend un sens différent que celui qui est le sien en français.

7 Le même Garner utilise le terme de ville ou de village indifféremment pour les ensembles de petite taille. Il est cependant difficile de mettre sur le même plan la cité de Noisiel, du chocolatier Menier (Valentin, 1994) et la ville-usine de Sedan à la même époque (Gayot, 1984), aussi bien pour des raisons de physionomie que pour le nombre d'habitants. Dans le premier cas, comme dans la plupart des villages industriels, la cité rassemblait un millier d'habitants au début du XXe siècle, autour d'une seule et unique 
entreprise, alors que dans le deuxième cas, on en comptait près de 20000 à qui une bonne dizaine d'industries de la laine donnaient du travail.

8 En Espagne, on utilise l'expression de " colonie industrielle » pour désigner ces villages ouvriers associés à leur usine, en chapelet le long des rivières qui fournissaient l'énergie. Comme il s'agissait de villages édifiés près des chutes d'eau mais loin des municipalités de référence, ils colonisaient un espace vide de toute construction, d'où le terme.

9 On peut se mettre d'accord sur le fait que le nombre des habitants et la diversité des services aident à caractériser un village industriel abouti, c'est-à-dire pleinement autonome. Trois exemples balayent le XIXe siècle, les deux premiers en Angleterre et le troisième en Italie : New Lanark (1800), Saltaire (1850) et Crespi d'Adda (1875). Ces trois villages industriels ${ }^{3}$ ont en commun des équipements et des services diversifiés autour de la culture, de l'éducation et de la santé. Leur nombre d'habitants a dépassé rarement les 2500 pour les deux premiers et les 1500 pour le troisième.

10 A côté de ces villages mono-actifs fortement structurés, il existe également des villes entièrement dédiées à une même industrie. Là encore le phénomène n'est pas nouveau en soi : au XVIIe siècle, Salins, en Franche-Comté, est une vraie ville-usine dont rendent compte quelques documents exceptionnels mais explicites : la saline est littéralement encerclée par la ville et la ville dominée par une série de fortins, car le sel est une marchandise précieuse; encore active au XVIIIe siècle, la ville-usine de la laine de Cerreto Sannita, en Campanie, (Rubino, G. 2018) distribue le travail dans chaque maison, l'élaboration finale du produit étant géré par la municipalité.

11 Ces structures issues de la proto-industrie vont être remplacées par d'autres, qui répondent à d'autres demandes du marché. Tout au long du XIXe siècle, des villes industrielles se développent, en assurant la gamme complète des activités relatives à une seule production : ainsi, Roubaix est la ville de la laine, mais aussi de la fabrication et de la réparation des machines et accessoires qui permettent la production. Saint Quentin fera de même, pour les cotonnades et les broderies à la machine. Les quartiers d'habitation se multiplient de façon anarchique, sur la base de courées, et se mêlent étroitement aux quartiers industriels. Roubaix et Saint Quentin sont aussi des villes donneuses d'ordres aux villages alentours qui travaillent pour elles, comme autant de villages ouvriers satellites.

Dans la deuxième moitié du XIXe siècle, le marché russe en pleine expansion suscite la formation de villes-usines industrielles de grande taille : Lodz en Pologne, de Tempere en Finlande, toutes deux textiles, dont on parlera plus loin. Elles sont à distinguer des villes mono-actives, entièrement consacrées à une seule et même entreprise, et elles aussi de taille gigantesque. Celles-ci sont nombreuses en Amérique du nord (Canada, États-Unis), au début du XXe siècle. Elles seraient plusieurs centaines dans la Russie soviétique, avec une chronologie décalée. Parmi celles qui sont édifiées dans les années 1930, on connait la ville de Magnitogorsk, dont la construction a fait l'objet de récits épiques (Scott, 1942). Parmi celles qui sont construites après la deuxième guerre mondiale il faut citer Togliatti, sur la Volga, une ville de 50000 habitants, dédiée à la construction automobile (Bellat, 2015).

Phénomène ancien, le village ouvrier ou la ville-usine sont de précieux indicateurs d'activité industrielle à un moment donné. Comme on l'a vu précédemment, elles correspondent à des étapes bien précises de l'industrialisation. Il ne s'agit pas cependant d'un développement in vitro, mais de grandes phases accompagnées 
d'événements politiques qui ont pu rejaillir sur leur création ou leur conception. Les débats utopistes qui ont agité les premières décennies du XIXe siècle, l'émergence de systèmes politiques conservateurs mais résolument industrialistes, les deux conflits mondiaux, ont eu leur impact et il est clair que le mouvement pour les cités-jardins en a bénéficié, comme on le verra plus tard.

Enfin, les désindustrialisations du dernier tiers du XXe siècle ont durement frappé les grands bassins industriels (Leboutte, 1997). Des villages, des villes-usines ont été désertés, ou continuent à être habités alors que l'usine, elle, a fermé. Les opérations d'aménagement du territoire ont parfois été bénéfiques à l'heure de donner une deuxième vie à un ensemble industriel dont la société civile ne voulait pas se séparer. Parfois la solution de l'arasement a été choisie, non sans créer des malaises et des contestations.

Il apparait donc utile de faire un détour par leur genèse et leur développement pour comprendre leur place dans le présent et les questions patrimoniales que ces structures soulèvent. D'autant que la perspective patrimoniale, loin d'être le résultat d'un choix réfléchi sur ce que l'on veut conserver et pourquoi, a toujours été précédée par un conflit de société. Aussi, plutôt que de revenir sur des sites connus et souvent mentionnés, nous avons préféré évoquer ceux qui ont une valeur exemplaire du fait de leur trajectoire et leur signification, sans pour autant appartenir aux pays les plus anciennement ou les plus densément industrialisés comme la Grande Bretagne (Edelblutte, 2010).

\section{I-Villages ouvriers et villes-usines de la première industrialisation}

16 Le village ouvrier, soit un groupe d'habitations, plus ou moins équipé, proche du lieu d'extraction ou de transformation, n'est pas une création récente. Les informations dont nous disposons dans l'espace européen, pour sporadiques qu'elles soient, sont indiscutables et datent de la plus haute antiquité (Della Monica, 1980).

Qui logeait-on et comment? Jusqu'au XVIIIe siècle, on loge les spécialistes, sans que ces logements soient de grande qualité : de simples et souvent petites chambres suffisent, sans point d'eau, mais avec un écoulement de l'évier à travers le mur, comme aux forges de Buffon, à Monbard (Rignault, 1978). Quant à la main d'œuvre, elle se loge dans les greniers quand ce n'est pas à la belle étoile. Le travail à domicile, d'une certaine façon, a compensé cette situation très inégalitaire en permettant que certaines étapes du travail ou certains types de fabrication qui demandent beaucoup de main d'œuvre, se fassent dans le cadre domestique. C'est le cas du filage et parfois du tissage à domicile, alors que l'ennoblissement du tissu se fait dans des manufactures ou des châteaux-usines comme le Dijonval de Sedan (Gayot, 1997). La structure d'Ancien Régime est bien là : les manufactures accueillent des gens de métier, parfois à grand frais. Ainsi, à Villeneuvette, près de Clermont L'Hérault, on fait venir des tisserands hollandais qui disposent d'une maisonnette et peuvent se réunir dans une chapelle à l'entrée de la cité. Ils vivent dans un espace délimité par les jardins qu'ils cultivent mais qui servent aussi à placer les rames pour blanchir les tissus. Au XVIIIe siècle, la cité dispose, comme dans d'autres manufactures à la même époque, d'un véritable parc, 
avec un buffet d'eau, signe que le métier exercé en ses murs est valorisé, et rentable (Thomson, 1982).

On peut estimer que le village ouvrier existe lorsque l'entrepreneur ou l'Etat (dans le cadre des manufactures) prévoit de loger sur le site de production non plus seulement les gens de métier mais aussi la plus grande partie de la main d'œuvre, à l'exception, éventuellement, de celle qui vient des villages voisins. Ce changement radical intervient dès la fin du XVIIe siècle dans les pays scandinaves (Ahnlund et Brunnstrom, 1992); au tout début du XVIIIe siècle, dans les villes-usines ouraliennes métallurgiques (Portal, 1950); en 1750 dans le cas de San Fernando de Henares, près de Madrid puis de la Manufacture de San Leucio (près de Naples) en 1789 (Dorel-Ferré, 2006), dont l'Edit du Bon Gouvernement associe pour la première fois de façon statutaire, le travail et logement. En cette fin du XVIIIe siècle, on trouve plusieurs villages ouvriers parfaitement constitués en Ecosse, en particulier ceux construits par le cotonnier Richard Dale, près de Glasgow (McLaren, 2016).

Un petit nombre de ces villages construits le long du XIXe siècle sont autant de jalons sur la route des villages créés par des patrons philanthropes, connus pour offrir à leurs habitants un niveau de services exceptionnel. Ils ont tous leur physionomie particulière, que ce soit Port Sunlight, près de Manchester, construit par le savonnier Lever ou que ce soit Cadbury, près de Birmingham, du chocolatier du même nom (Dorel-Ferré, 2016). Sur le continent, Le Grand Hornu, près de Mons en Belgique wallonne (Watelet, 1993), est un site minier et métallurgique unique en son genre. La cité ouvrière, composé de maisons accolées, offre en 1830 un niveau de confort inouï, avec de grandes pièces différenciées pour les parents et les enfants et l'eau chaude dans chaque maison (fig. 1).

Figure 1 : Grand Hornu

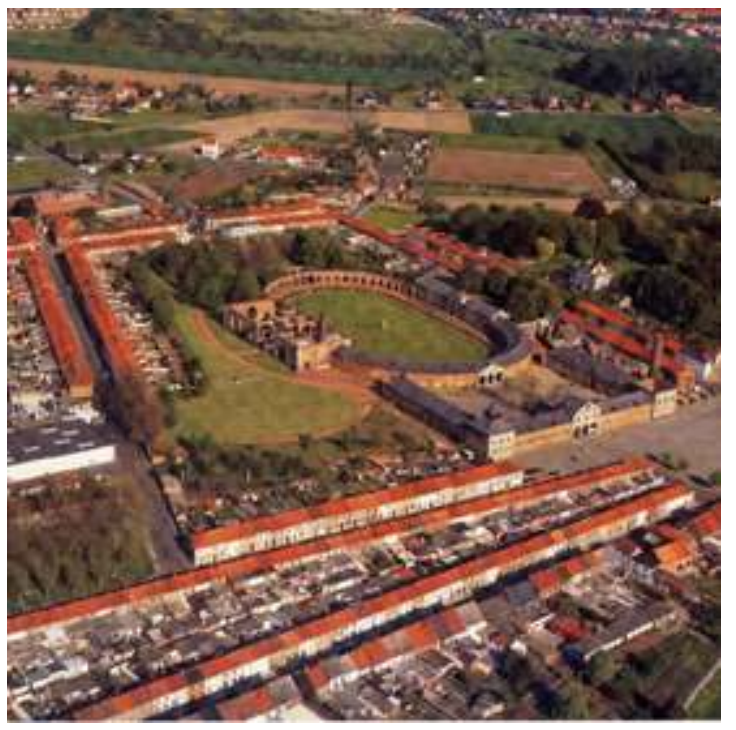

Photo : Grand Hornu Images

En 1867, Lorsque Napoléon III décide que l'Exposition Universelle de Paris portera sur le logement ouvrier, il n'existe que peu de villages semblables en France, hors la cité Dollfus à Mulhouse (Jonas, 1994) ${ }^{4}$. Godin, qui n'a pas terminé son Palais social de Guise (il ne le sera pas avant 1870), a dédaigné concourir. En 1873, lorsque Engels fait le tour 
de la question dans trois articles destinés à la Gazette de Leipzig, il souligne le piteux état de la construction des logements ouvriers dont le seul but semble être le moyen, par le propriétaire, de récupérer une bonne partie du salaire versé. Pour voir émerger une vraie préoccupation et de vraies solutions à cette «question du logement » qui traverse le XIXe siècle, il faut attendre l'Exposition Universelle de Paris de 1889 et les rencontres institutionnelles qui l'on suivie ${ }^{5}$.

21 Si nous limitons notre étude à l'espace européen occidental, nous pouvons estimer que le XIXe siècle, jusqu'au tournant des années 1875 , est celui des réalisations aussi spectaculaires que peu nombreuses et isolées. Par contre, avec la croissance du dernier quart du XIXe siècle, l'économie s'emballe et les constructions industrielles se multiplient en fonction des sources d'énergie et des ressources du sous-sol, dans une inscription spatiale distincte. Ainsi, nous voyons la formation de grappes de villages ouvriers, le long des cours d'eau, structurés en systèmes territoriaux plus ou moins aboutis. Ce seraient, par exemple, les villages de la soie des Cévennes, dont Nîmes et Lyon organisent la production, les villages textiles des Vosges, tournés vers Epinal après 1871, ou encore les "colonies industrielles catalanes", étroitement liées à Barcelone qui leur livre le coton brut et commercialise les textiles teints et finis (DorelFerré, 2017a). En même temps les bassins de charbon et de ressources métalliques diverses (fer, cuivre, etc.) se développent et répandent leurs cités ouvrières là où le terrain ne comporte pas d'enjeux, mais à une proximité relative du lieu de travail (Le Maner,1995).

Qu'en est-il des sites dont l'activité a traversé les siècles, et qui par conséquent n'ont pas fait l'objet de constructions planifiées comme dans les exemples précédents ? Peu d'entre eux datent de l'Antiquité, comme Almadén (Espagne), ou du Moyen-Age comme Salins (Franche-Comté) ou encore Wieliczka en Pologne. Certains ont succédé à une manufacture royale ou privilégiée comme Saint Gobain, par ailleurs lié à une ressource locale indispensable comme le sable et le bois pour les verriers ou les céramistes. Leur expansion est liée à un marché porteur : le mercure, le sel, le verre. L'analyse montre qu'ils ont subi deux vagues d'interventions décisives, l'une au XVIIIe siècle, dans l'effort général pour rationaliser les conditions de la production, l'autre au XXe siècle, pour rationaliser la place de la main d'œuvre.

Almadén, la ville du mercure sur le flanc nord de la Sierra Morena, en Espagne (Chastagnaret, 2002, Dorel-Ferré, 2012), est connue depuis l'Antiquité romaine puisqu'on y récoltait le rouge vermillon qui était employé dans les fresques murales comme à Pompeï. Les musulmans l'ont exploité et le vocabulaire relatif à la mine et à son exploitation date de leur passage. La ville s'est développée sur le promontoire qui chapeaute la mine, dans une imbrication étroite. Mais son expansion date surtout du XVI siècle, lorsque est mis au point le procédé d'extraction de l'argent par amalgamation avec le mercure : à partir de ce moment-là, un effort considérable est fait pour augmenter la production que l'on envoie en Amérique, d'où reviendra l'argent de Potosi et autres lieux. Le banquier Fugger obtient les revenus d'Almaden en garantie des prêts concédés à Charles Quint, et pour accélérer la production, introduit les esclaves parmi les ouvriers. Ils constitueront $20 \%$ de la main d'œuvre jusqu'au XIXe siècle. Cependant, les ouvriers spécialistes ne se pressent pas dans cet univers très dur, aux maladies professionnelles incurables. Pour les attirer, Villegas, administrateur des mines au XVIIIe siècle, met en place un programme social et culturel, à la fois original et rentable, qui assure à la fois le logement, les loisirs et la santé. 

taureaux sont construits, au pied de la ville. Non loin, un hôpital doit accueillir les malades atteints d'hydrargie. Les frais engendrés par les logements et l'hôpital sont couverts par les revenus des courses de taureaux. De plus, chaque ouvrier dispose chaque année, par tirage au sort, d'un lopin dans la Dehesa, la campagne environnante, afin de respirer au grand air et s'adonner à la chasse, la pêche et le loisir. Dans le même temps, pour maîtriser la population esclave, un grand bâtiment est construit à son intention, comme une caserne fortifiée, d'où les forçats ne sortent que pour se rendre dans la mine où les attend le travail pénible et incessant de l'exhaure. Au XIXe siècle, le travail des forçats est aboli. Cependant la grande caserne accueille en 1939 les prisonniers républicains qui seront traités comme leurs prédécesseurs. Le bâtiment, trop chargé d'images négatives, est rasé en 1970. Le site minier a été fermé récemment et a fait l'objet d'un classement au patrimoine mondial depuis 2013. plus remarquable, servi par une longue histoire. Sans doute exploitée depuis les temps préhistoriques, la saline de Salins fournit une bonne part des subsides sur lesquels s'appuie la dynastie des princes de Bourgogne, aux XIVe et XVe siècle. Du fait de la valeur stratégique et économique du sel, la saline était incluse dans la ville qui la protégeait et défendue par des forteresses dressées sur les montagnes environnantes, qui surveillaient les allées et venues dans la vallée. Cependant l'inconvénient d'aller toujours plus loin pour se procurer le bois de chauffage nécessaire avait fini par rendre la production coûteuse et peu rationnelle. Ledoux, architecte auquel Louis XV confie la direction des salines décide de transporter l'usine au milieu de la forêt plutôt que de « découper celle-ci en briquettes » selon ses propres paroles. C'est le point de départ de la manufacture d'Arc et Senans (1774-1776), inachevée, mais dont l'architecture néoclassique et le programme social sous-jacent a fait couler beaucoup d'encre. Certains y ont vu une cité utopique, encouragés en ce sens par l'auteur lui-même qui décrit son œuvre un quart de siècle plus tard (Ledoux, 1804). Rien n'est plus contestable ; tout au contraire de Salins, qui est une ville-usine, Arc-et-Senans est une manufacture royale, qui loge les spécialistes, pas la main d'œuvre. Cette dernière venait des villages environnants. La production est rationnalisée et l'eau salée produite à Salins est expédiée à Arc et Senans par saumoduc. L'ensemble n'aura pas le succès escompté, et la forêt reste surexploitée, jusqu'à une époque récente. (Boully, 2013 ; Scachetti, 2013.)

Il en va autrement de la glacerie de Saint Gobain, fondée à la fin du XVIIe siècle, où vivent les maîtres verriers et leurs familles, en permanente compétition avec les habitants privilégiés de la ville pour l'exploitation de la forêt (Hamon, 1993). La nuit du 4 août 1789 et l'abolition des privilèges privent la ville de ses droits forestiers et la met entre les mains du directeur de la glacerie, seul pourvoyeur d'emplois désormais. Au cours du XIXe siècle, la ville devient le village ouvrier de la manufacture et les quartiers ouvriers se multiplient, sans plan particulier sinon celui de la faisabilité, au rythme de son expansion. Lorsque la compagnie quitte le site éponyme en 1995, elle laisse la ville orpheline, avec un patrimoine bâti exceptionnel, que la municipalité entend aujourd'hui mettre en valeur (Bliaux, 2019).

Almadén, Salins, Saint Gobain, sont incontestablement des villes-usines, qui se sont formées, le long des siècles, sur leur propre lieu de production jusqu'à se fondre avec lui. On pourrait faire une analyse comparable pour Le Creusot, né à la fin du XVIIIe siècle, dont le plan incohérent est celui d'une ville dont l'expansion s'est faite, comme

Revue Géographique de l'Est, vol. 58/3-4 | 2018 
Saint Gobain, par à-coups (Bergeron, 1995), ou encore de Clermont-Ferrand, la Michelin-ville (Gueslin, 1994). Le Creusot et Clermont-Ferrand sont deux visages d'un même phénomène: celui d'une ville patronale, d'une seule et même famille. A une échelle, autre que les villages industriels mais pas différents de nature.

Cependant à côté de ces villages ouvriers et de ces villes industrielles anciennes, il se développe des villes que l'on peut classer dans deux groupes différents, la ville-usine non planifiée et la ville-usine planifiée (Edelblutte, 2010).

Les villes-usines non planifiées se forment dans le dernier tiers du XIXe siècle. Composées d'agrégats d'usines gigantesques, produisant dans la même branche, elles associent dans un espace contigu un tissu d'habitat parfois désordonné, mais aussi des cités ouvrières et leurs maisons patronales. Deux exemples : Lodz (Anikinow, 2005) en Pologne et Tempere en Finlande, toutes deux villes champignons du textile de coton, toutes deux dynamisées par la proximité de l'immense marché russe.

Lodz est un hameau au milieu de XIXe siècle lorsque des ingénieurs allemands s'associent à des nobles polonais et à des juifs qui apportent les capitaux, pour implanter une industrie textile. On y produira le coton pour Saint Pétersbourg et le marché russe, en plein développement ! En 1914, la ville compte 47 usines et occupe plus de 500000 habitants, avec une forte proportion d'allemands et de juifs. Parmi ces usines, les plus énormes sont celles de Poznanski, aujourd'hui transformées en centre commercial et de Schleiber, avec sa cité ouvrière modèle du Moulin du Curé.

31 Tempere n'a pas cette taille mais la même trajectoire. Là ce sont des écossais, attirés par le marché russe, qui ont implanté des usines textiles, en bénéficiant d'une énergie hydraulique puissante. Il n'y a pas à Tempere le luxe ostentatoire des industriels polonais. Nous sommes en pays protestant. La main d'œuvre s'était entassée dans des conditions insalubres révoltantes. La ville décide de planifier, autour des usines, un quartier ouvrier, Amuri, en damiers réguliers, chaque carré se composant d'une cour entourée de logements, construits en bois, avec une cuisine commune pour deux à quatre familles. Les logements sont très sommaires : un lit, parfois une petite table et dans le meilleur des cas, une commode. Dans la cour, les saunas collectifs et éventuellement une coopérative d'achat. Dans les années 1970, ce quartier a été rasé et remplacé par des immeubles d'habitation. Le plan général seul subsiste ainsi qu'un pâté de maisons, conservé et converti en musée (Keijo Rantanen, 2010).

\section{II-Villes-usines et cité-jardin de la deuxième industrialisation}

32 La ville-usine du début du XXe siècle n'a toujours pas résolu la question du logement. Les enquêtes, nombreuses, faites sur les conditions de vie des ouvriers dans les villages et cités ouvrières pointent les limites du système: Owen, le créateur de l'expérimentation sociale à New Lanark avait été obligé de partir, en 1825 sous la pression de son conseil d'administration, qui estimait que l'argent consacré à la politique sociale était de l'argent perdu ; à l'autre bout du siècle, Zola décrit dans ses Carnets les tares du «Palais social » de Godin, sclérosé par un comité de gestion frileux ; l'écrivain Remont dénonce, en 1899 les méfaits de l'industrialisation à Lodz dans un roman qui sera un grand succès du cinéma sous le titre La terre de la grande promesse, d'Andrzej Wajda. La généralisation des maisons mitoyennes en ligne ou corons, sans 
compter les nombreuses courées, les "closes» et autres "conventillos" attirent les critiques mais aussi de nouvelles propositions de la part des architectes ${ }^{6}$.

En 1898, Ebenezer Howard propose alors un concept vite dévoyé mais au succès incontestable : the city-gardens for to-morrow, qu'il complète en 1902. On retient l'idée d'un espace plus ouvert, au plan sinueux, boisé, protégeant la vie privée. C'est la citéjardin contre le coron, du moins c'est ainsi que le présente une publicité de l'époque. Peu de réalisations voient le jour, cependant, avant la Première guerre mondiale. Par contre, au lendemain du conflit, avec l'urgence de la Reconstruction, une prolifération impressionnante d'initiatives font de cette première partie du XXe siècle, le siècle des villages ouvriers de grande taille et des nombreuses villes-usines, où sont généralement adoptés les principes de la cité-jardin. L'unité de base est la maison mitoyenne par deux ou par quatre, inspirée de la maison mulhousienne, le long de rues courbes qui évitent les vis-à-vis directs, avec une place centrale où se dressent les habituels lieux de pouvoir : l'église, la mairie, éventuellement la maison du directeur si la cité-jardin est d'initiative patronale. Ces cités nouvelles sont assorties de programmes sociaux, culturels et sanitaires qui paraissent désormais indispensables. Cependant, les plans très variés montrent vite des inspirations différentes. Deux grandes familles se distinguent: une vision géométrique de l'espace, illustrée ici par les exemples hongrois ; la vision jardinée et sinueuse, dont on retiendra l'exemple français.

Les deux exemples hongrois, nous éclairent sur le passage de l'un à l'autre : Diozsgör (Olajos,1998) dans le Nord-Est près de Miskolcz, et la cité-jardin de Werkelé (Nagy, 1994, Jonas, 2004) dans Budapest ; la première (fig. 2) est une cité patronale, commencée dans les années 1870. Les plans de 1910 présentent une grande uniformité de maisons jumelles disposées le long des rues avec chacune un lopin tête-bêche avec le lopin de la maison parallèle. Mais il existe aussi des maisons plus larges et mieux dotées, pour les cadres et les employés. Plus tard, dans les années 30, on construit à leur intention de petits immeubles d'appartements. De nombreux services sont proposés : école maternelle, écoles primaires ; caisse d'assurances ; hôpital, département de gynécologie et maternité, foyer des infirmières, pharmacie. A partir des années 20 , on ajoute une poste, une gendarmerie, des bains publics, des magasins d'alimentation, une boucherie, un restaurant, et un marché couvert. Du point de vue confessionnel, on trouve une église catholique, une église évangélique et un temple réformé. Dès la fin du XIXe siècle, le village est équipé d'eau potable, de tout à l'égout et un peu plus tard, de réseaux électriques. Cependant, le plan, très géométrique, ne protège pas des vis-à-vis et les espaces verts sont peu nombreux. C'est un exemple parfait du plan orthogonal. 
Figure 2 : Diozsgör en 1910

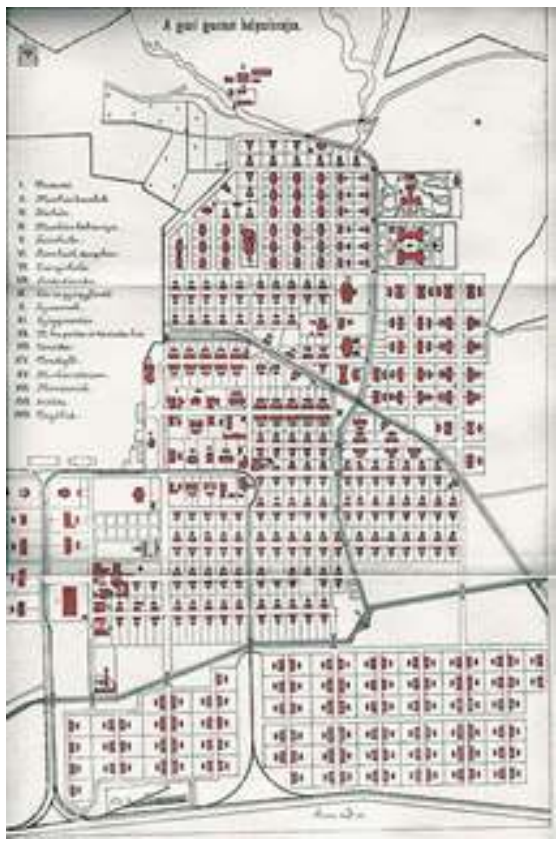

Csaba, 1998

Wekerlé (fig. 3) date des années 1920. C'est un village pour ouvriers et employés, et il sera tardivement intégré à la ville de Budapest. L'espace très boisé compense le plan géométrique, en quadrilatère, autour d'une place centrale. Bien que les maisons aient été construites avec des matériaux de qualité moyenne, et leurs surfaces très modestes, le site s'est plutôt bien conservé. Une certaine ambiance de village a perduré, qui le rend aujourd'hui tout à fait attractif.

Figure 3 : Le plan de Wekerlé en 1926

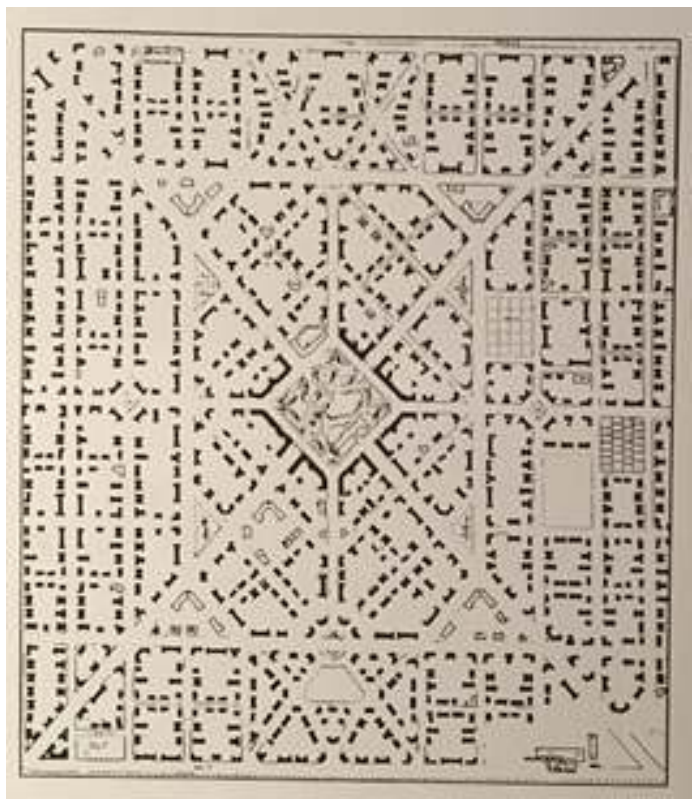

Nagy, s.d 
France, les réalisations seront nombreuses au cours des années 20, depuis des cités urbaines comme Suresnes jusqu'à la cité emblématique de Chemin Vert à Reims, œuvre de l'industriel philanthrope Georges Charbonneaux (Dorel-Ferré, 2016). Elles se font dans le cadre de la Reconstruction des régions dévastées, assorties d'un programme social rendu nécessaire par les désastres de la guerre L'ensemble le plus impressionnant reste celui des cités ferroviaires de la Compagnie du Nord, qui appartenait à Rothschild, et dont la direction avait été confiée au polytechnicien Raoul Dautry. Ce dernier veut aller vite : il faut que les communications soient rétablies entre Paris et le bassin minier, tout en évitant que les populations meurtries par la guerre ne se jettent dans les bras du communisme. Il décide donc la création de cités ferroviaires, sur tout le territoire de la compagnie, dont la plus grande, Tergnier, concentrait plus de 2000 habitants (fig. 4). Alors que les autres cités ferroviaires de la compagnie adoptent un plan sinueux propre aux cité-jardin, à Tergnier, le plan prend la forme de trois roues d'une locomotive et la roue centrale concentre tous les services. Les maisons de la Compagnie déclinent un module de base où la chambre des parents séparée de celle des enfants, la cuisine avec l'eau sur l'évier, la salle de bain et les commodités sont prévues pour tous ; dans les maisons de cadre, la salle à manger est distincte, le salon est prévu pour contenir des fauteuils et canapés, la salle de bain comporte une vraie baignoire. Ces dispositions vont de pair avec une politique hygiéniste très complète, des loisirs sportifs, une vie culturelle intense où le journal et la chorale tiennent une grande place (Dorel-Ferré, 2017b).

Figure $4:$ La cité de Tergnier

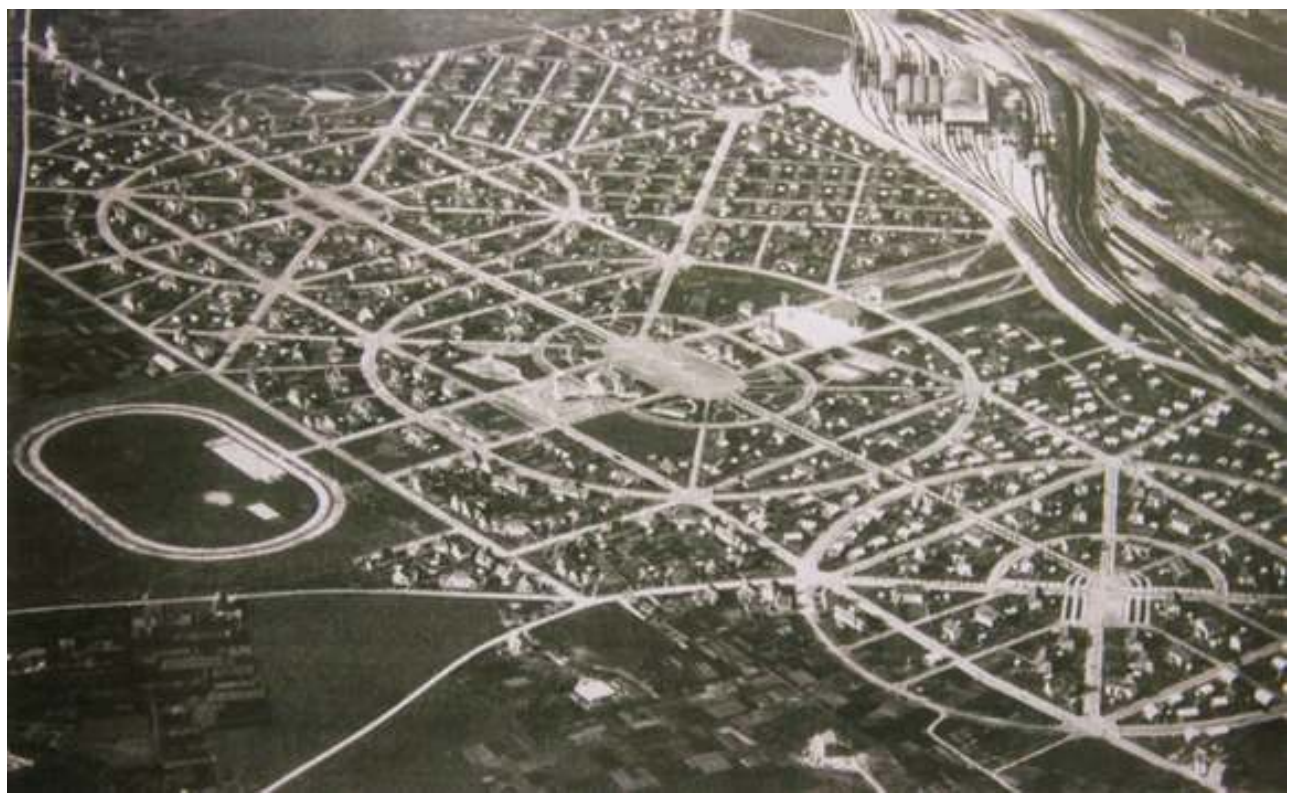

Archives SNCF

Dans le cas des villages ouvriers déjà formés à la fin du XIXe siècle et agrandis dans la première moitié du XXe siècle, on peut parler de villages mixtes. En effet, à côté des infrastructures anciennes, antérieures à 1900, s'ajoute une extension inspirée de la cité jardin, avec des rues courbes, des espaces verts, une infrastructure sociale visible, à travers des monuments qui sont autant de repères nouveaux: école, dispensaire, 
bibliothèque, etc. C'est le cas de Crespi d'Adda près de Bergame, en Italie, ou encore de Solvay à Salin de Giraud (Daumalin et al., 2012).

Dans les années 1930, dans le contexte de l'Art Déco, la réflexion qu'apporte le Bauhaus et que reprend Le Corbusier pour la France, donne un ton nouveau, dans lequel s'insèrent des créations comme Bataville en Lorraine.

39 Les régimes autoritaires s'emparent de la formule, dans le cadre d'une politique d'Etat au service des grands intérêts privés, comme ce fut le cas de l'Italie fasciste, avec des structures à mi-chemin entre le village ouvrier, dont elles ont la taille, et la ville usine, dont elles ont l'ambition. C'est le cas entre autres de Dalmine, près de Milan, pour les constructions mécaniques (Lussana, 2003) ou de Valdagno (Fontana, 2003), près de Padoue, pour le textile. Plus tard, l'Espagne franquiste prendra le relai, avec son grand programme de villages ouvriers agricoles ou industriels. Tard venu, ce programme aura été vite déclassé par les grandes désindustrialisations du dernier tiers du XXe siècle et n'a pas suscité jusqu'à présent, d'études de synthèses (Alvarez Areces, 2015).

Dans l'Europe médiane, de la Belgique à l'Allemagne rhénane des années 20 , on a pu parler d'un modèle germanique (Jonas, 2004). Joint aux recherches du Bauhaus, a-t-il inspiré les architectes de la Russie soviétique? C'est en effet la période des grandes réalisations constructivistes dans les plus grandes villes et en particulier à Ekaterinbourg où la cité des Tchékistes est un double de la cité-jardin de Suresnes. Ce sont les débuts des villes géantes surgies du néant, comme, au sud de l'Oural, Magnitogorsk (fig. 5), déjà citée (Scott, 1942). Disposant d'un espace quasiment infini, ces villes acquièrent une physionomie singulière. Une nouvelle distribution urbaine apparaît, avec de larges avenues articulées autour de la Maison de la Culture, qui trône devant une large place, accompagnée éventuellement par la Mairie et l'Université. Les différents carrefours accueillent les autres repères civiques : le dispensaire, l'hôpital, la bibliothèque et le théâtre s'il y a lieu, mais aussi la piscine et le stade, à l'entrée monumentale. Les logements se font par groupes d'immeubles, dans des écrins de verdure qui sont investis à la belle saison par les gens du quartier.

Figure 5 : Magnitogorsk

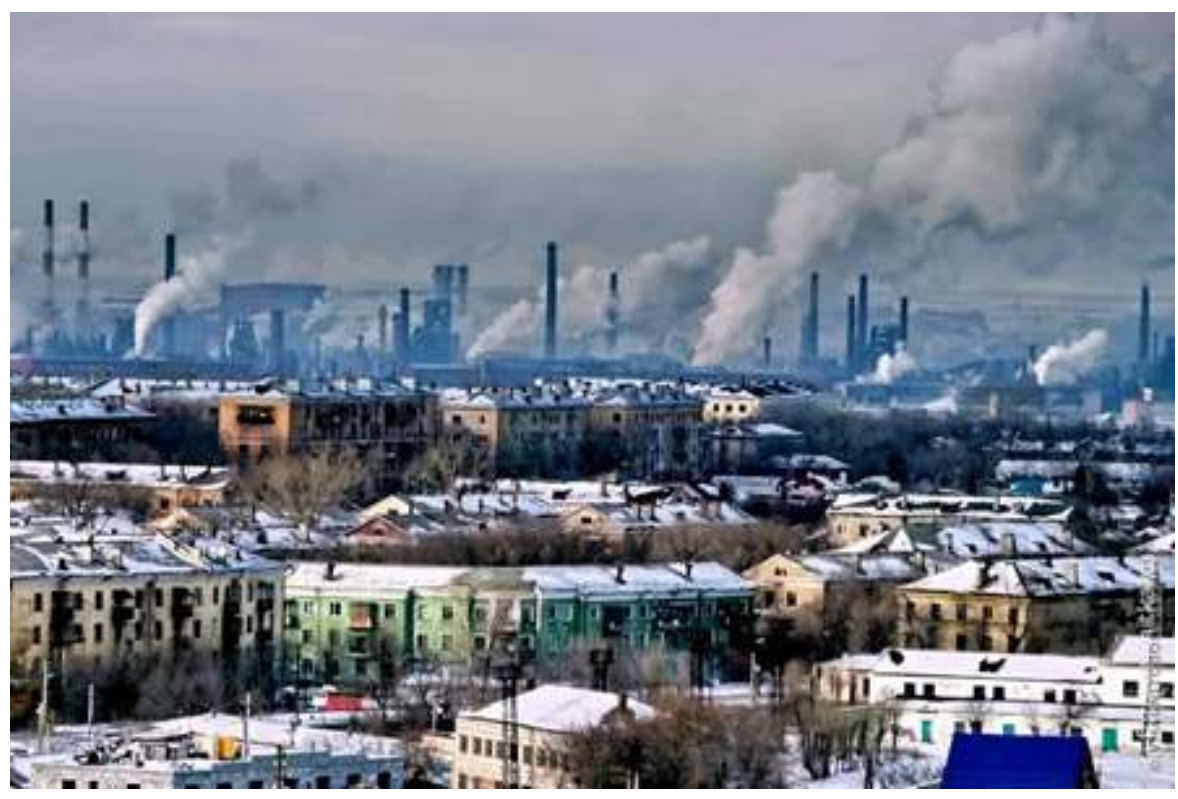

Cliché APIC

Revue Géographique de l'Est, vol. 58/3-4 | 2018 
41 lieux collectifs (théâtre, église, maison de direction), des services nombreux (commerces, marché couvert, piscine, loisirs, santé) une certaine ambition dans des logements de qualité et leur vocation sociale, largement affirmée.

Après la deuxième guerre mondiale, alors que les pays occidentaux abandonnent l'idée de la cité-jardin parce que trop coûteuse, la Russie soviétique lance un programme nouveau : on compte environ 2000 villes nouvelles, disséminées sur le territoire russe, dont Togliatti, (Bellat, 2015). Comme pour les villages ouvriers, les villes-usines anciennes, nées au XVIIIe siècle, près du barrage qui leur fournissait leur énergie, connaissent une période d'aménagement et d'extension. C'est le cas de Nijni Taguil et d'Ekaterinbourg en Oural. Si pour Magnitogorsk on était allé au plus pressé, construire des logements, mais sans penser à l'infrastructure qui viendrait après, les nouveaux quartiers de Nijni Taguil sont prévus dans leur globalité, comme le réclamait déjà l'architecte Guinzbourg en 1935. On retrouve les préoccupations des années 30, que l'on soit à l'Est ou à l'Ouest: une architecture arborée, des services sociaux diversifiés. La nouvelle ville d'Ouralmash (fig.6), nouveau quartier d'Ekaterinbourg de 50000 habitants, sera construite sur ces principes. En Pologne, la «nouvelle forge » près de Cracovie ou Nowa Hutta développe un plan et une structure du même type: une organisation très géométrique, avec un système de rues en patte d'oie, mais très arborée, les immeubles disséminés dans la verdure avec à proximité, une desserte économique (magasins, restaurants), culturelle (kiosques à journaux, bibliothèques) et sociale (dispensaires) visible...

Figure 6 : Ouralmash en 1958

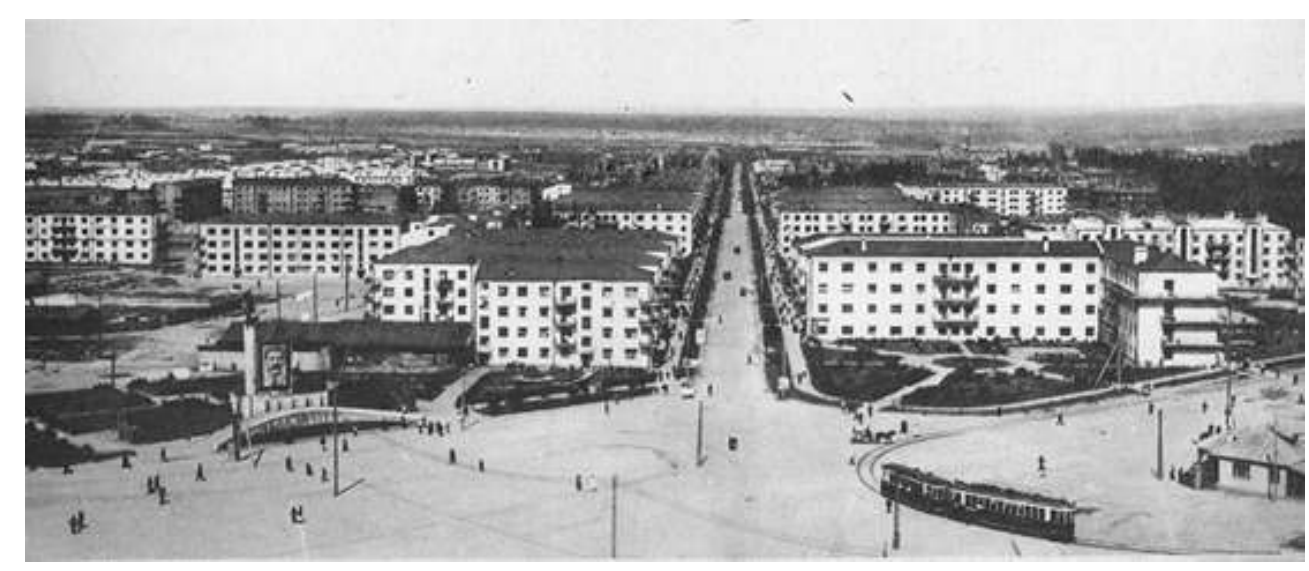

Archives locales

Pour la plupart de ces villes-usines d'Europe se pose le problème de leur survie ou de leur adaptation à une raison d'être différente: conçues comme habitat d'élite à proximité de l'usine gigantesque qui pourvoyait à tout, elles doivent apprendre à vivre sans ce qui était leur centre de décision et leur ultime recours. Certaines sont tout simplement abandonnées. Pour beaucoup d'entre elles, la patrimonialisation est le moyen de perdurer. 


\section{III-La patrimonialisation, un remède quand l'usine a fermé ?}

Depuis le dernier tiers du XXe siècle, il ne s'agit plus de loger systématiquement les ouvriers et l'encadrement près du lieu de travail, bien au contraire, sauf dans les situations extrêmes (extraction de minerais à haute altitude, ou en limite climatique, ou encore en mer sur les plateformes pétrolières). Les entreprises, aujourd'hui, répugnent à se charger d'une gestion qui, leur semble-t-il, n'entre pas dans leur compétence; les moyens de déplacement individuels et collectifs permettent d'habiter là où l'on souhaite. De ce fait, la relation directe entre usine et lieu de vie est rompue, ou du moins elle n'est plus apparente.

D'autant que deux phénomènes étroitement liés sont intervenus qui ont radicalement transformé le contexte économique et social : à partir des années 1970 et en moins d'un demi-siècle, les grandes désindustrialisations prennent l'Europe en écharpe, des Iles Britanniques à l'Oural. Parallèlement, les activités qui ont remplacé les industries disparues changent de nature : la robotisation implique moins d'agents et introduit une distance entre l'ouvrier et la machine ; les activités tertiaires prennent de plus en plus de place et induisent des images du travail différentes: le bureau et l'ordinateur remplacent la machine Les grandes concentrations industrielles des années 1950 font bientôt partie d'un passé révolu.

Cette évolution étant prévisible, certaines entreprises ont anticipé la fin en décidant leur mise en patrimoine. C'est le cas de Charbonnages de France, qui sachant la fermeture prochaine, ont choisi un lieu, la Fosse Delloye, à Lewarde, près de Douai, pour en faire un musée de la mine qui accueillerait les archives du groupe et une recréation de la mine, présentée par d'anciens mineurs. On sait que l'ouverture du musée en 1984, coïncidant avec la sortie du film Germinal de Claude Berri avait provoqué une véritable affluence. Prévu pour 70000 visiteurs, le musée en accueillit bientôt le double. Quant aux structures plus ou moins désaffectées du Bassin minier (cités, carreaux de mines, chevalements, équipement divers) il leur fallut attendre et se battre pour une reconnaissance et la promesse d'une mise en valeur pendant longtemps avant d'obtenir, en 2012, l'inscription sur la liste du patrimoine mondial de tout le Bassin minier, sans que cette protection soit toujours parfaitement efficace. Si les bâtiments d'administration vastes et bien construits, peuvent être reconvertis en services régionaux et universités publiques, les immenses cités ouvrières reconstruites après la Première guerre mondiale peinent à trouver une autre affectation. Différents facteurs interviennent : l'éloignement des centres de services et de loisirs, le manque d'activités et de perspectives sur place, la mise en tourisme pas toujours efficace...

Le cas de Chemin Vert, à Reims, mentionné plus haut, est assez révélateur. Réalisé dans les années 20 pour offrir des logements à loyer très faible pour familles nombreuses, cette cité-jardin avait bénéficié de l'aide d'architectes et d'artistes de premier plan. Mais dans les années 1980, c'était devenu le type même du village abandonné, triste et sale. Il a bénéficié d'une restauration soignée depuis les années 1990, et aujourd'hui, vivre à Reims dans ce qui a gardé le cachet d'un joli village fleuri, est assez recherché. C'est qu'avec la croissance urbaine, Chemin Vert, qui avait été édifié à l'extérieur de la ville est maintenant en son centre. Sa fonction habitat a été réévaluée et sa physionomie soigneusement conservée puisqu'il s'agit, depuis l'origine, d'un habitat en location. Deux monuments s'élèvent sur la place : la «maison commune » qui faisait 
office de mairie annexe, et regroupait la bibliothèque, la salle de réunion, le théâtre et les douches. L'église est un petit bijou, où sont intervenus le peintre Maurice Denis, le maitre verrier Lalique, et d'autres. Pourtant, la mise en tourisme de cet ensemble exceptionnel tarde à se concrétiser, sans doute parce que la compétition touristique que livre la cathédrale de Reims est écrasante.

Tourisme et patrimoine ne sont donc pas une formule valable à tous les coups, et les facteurs de réussite sont parfois bien mystérieux. Les colonies industrielles catalanes sont, dans cette optique, un cas d'école, dont nous rappelons les principaux traits :

Dans la Catalogne intérieure, sur le cours moyen des fleuves Ter et Llobregat qui fournissent l'énergie, on compte près de 90 villages industriels, construits pour la plupart entre 1880 et 1905, de taille diverse, depuis l'isolat qui ne comprend que l'usine et quelques logements jusqu'au village le plus développé comprenant l'usine, la maison de maître, l'église, l'économat et les logements en immeubles ou en maisons mitoyennes. C'est un paysage exceptionnel, car les colonies s'égrènent tous les kilomètres, kilomètres et demi, formant une rue d'usine depuis les Pré-Pyrénées jusque près de la capitale. La désindustrialisation du dernier tiers du XXe siècle a conduit à fermer toutes les usines, sans exception. Certaines colonies ont été abandonnées par leurs habitants, mais la plupart sont toujours habitées.

Deux colonies se détachent du lot : la plus importante, la Colonia Sedó (fig. 7), qui se trouve à une quarantaine de kms de Barcelone et la plus connue, la Colonia Guëll, dans l'aire métropolitaine.

51 La Colonia Sedó, du fait d'un système hydraulique exceptionnel, a été la plus grande des colonies industrielles catalanes. En effet, le cours du Llobregat a été barré $4 \mathrm{kms}$ en amont pour alimenter, par une conduite forcée en partie souterraine, en partie en aqueduc, un jeu de turbines remplacé en 1899 par une seule, de $1400 \mathrm{CV}$, la plus grosse jamais construite en Espagne. Grâce à cette installation unique en son genre, l'usine a pu accueillir jusqu'à 2250 ouvriers dans les années 1920, alors que la plupart des autres colonies industrielles n'en rassemblaient pas 500. Le village industriel, dominé par son église et sa maison de maître, logeait une partie des familles, environ $20 \%$ de la main d'œuvre. L'usine a fermé ses portes en 1979.

Un musée est installé sur les lieux de la turbine que sa taille gigantesque rendait inutilisable. On peut aujourd'hui la parcourir de l'intérieur et visiter les deux niveaux d'installations hydrauliques : celui qui a fonctionné entre 1878 et 1899, et celui qui a été mis en place, par-dessus le précédent, en 1899. C'est un témoignage unique de l'extraordinaire importance de l'énergie hydraulique dans un pays qui était dépourvu de toute ressource minière et minérale. 
Figure 7: La colonia Sedo d'Esparreguera

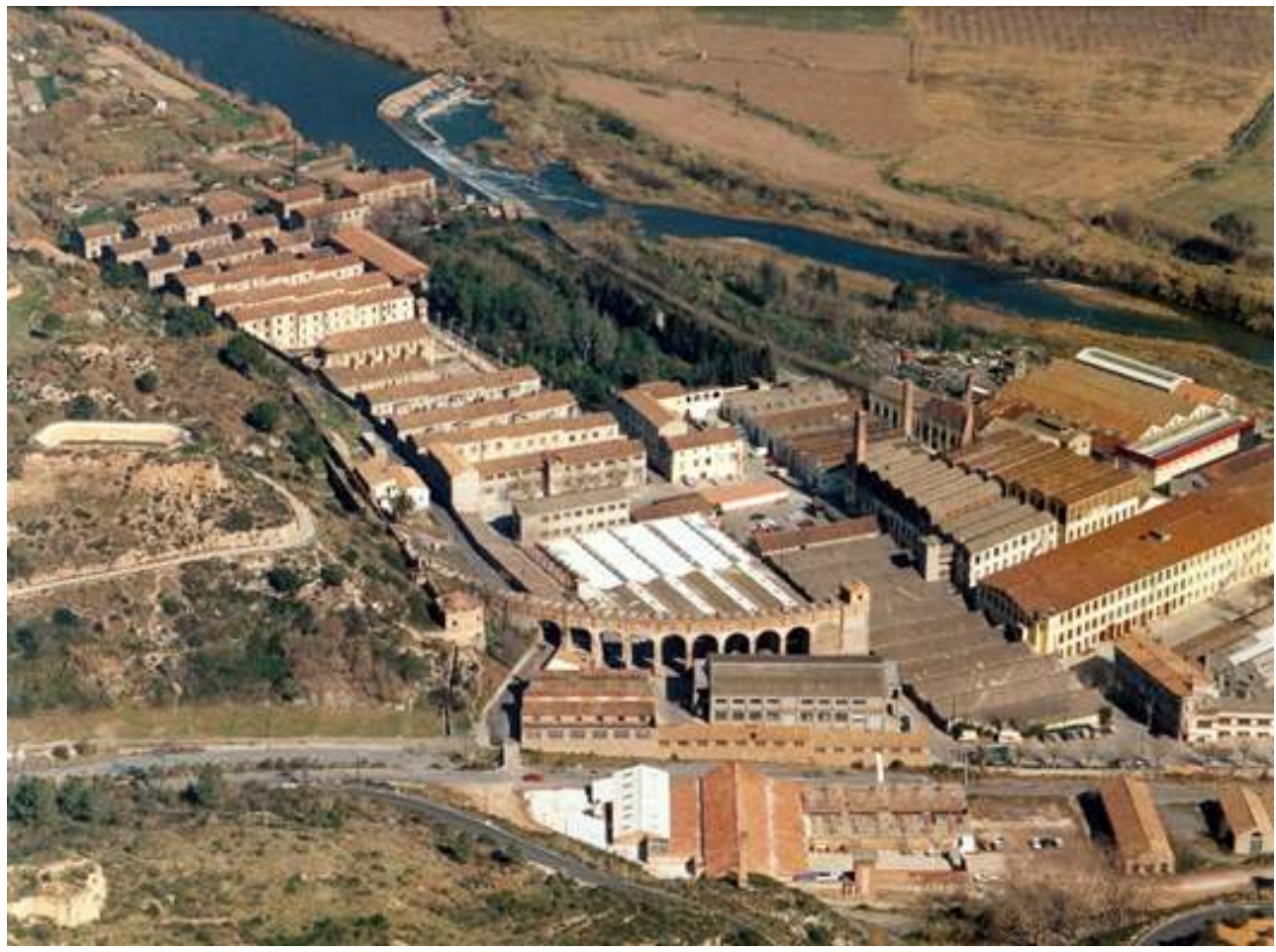

Photo Municipalité d'Esparreguera

Tout récemment qu'un projet d'aménagement global a été pris en compte par la municipalité. Déjà des circuits sont proposés à l'intérieur du polygone industriel comme à l'extérieur; l'ancienne église acquiert progressivement le statut de centre culturel ; des espaces sont réservés aux activités scolaires ; un projet d'aménagement de l'ancienne maison des maîtres prévoit une auberge de jeunesse et des hébergements de charme, près du grand parc. C'est à un vrai pôle d'activités culturelles et de loisirs est en train de se dessiner, à mi-chemin entre Barcelone, et ses quatre millions et demi d'habitants et le monastère de Montserrat qui accueille tous les ans deux millions et demi de visiteurs.

La Colonia Guëll, de taille modeste, est la seule de toutes les colonies textiles à fonctionner à la vapeur. Elle est née du projet d'Eusebi Guell, dont on ne parlerait sans doute pas aujourd'hui s'il n'avait fait appel à l'architecte catalan le plus emblématique : Gaudi. La Colonia Guëll a été inscrite au patrimoine mondial avec l'ensemble des œuvres de Gaudi. Proche et accessible depuis Barcelone, elle est la colonie industrielle la plus visitée.

Trois solutions, donc, se présentent aux villages et villes-usines, lorsque toute activité a cessé : l'abandon pur et simple, la fonction résidence et la fonction tourisme. On peut y ajouter une quatrième : la reconversion pour d'autres usages (Gasnier, 2018).

La fonction tourisme et la conversion vers d'autres activités est complexe, car il s'agit d'un projet qui engage la société non pas dans l'usage du présent mais dans un choix de futur. Or les résultats peuvent être très différents, sans que l'on puisse vraiment agir sur les causes : aux 450000 visiteurs de New Lanark, près de Glasgow, on peut opposer le faible nombre de visiteurs de Crespi d'Adda, non loin de Bergame. Il est vrai que le site 
de New Lanark sur les bords de la Clyde est un lieu de promenade aimé et connu des gens de Glasgow, alors que le site de Crespi est isolé ....

Dans tous les cas, la reconversion du patrimoine n'est pas portée par des spécialistes du patrimoine, qui jouent éventuellement le rôle de conseils, mais par la société ou un groupe influent. Ceux-ci ne reprennent ces sites que pour les transformer et les adapter à leur usage, parfois bien loin de la situation initiale. Ainsi, le groupe Nestlé a réhabilité le site de Noisiel, du chocolatier Menier, d'une façon exemplaire, mais en le coupant de la cité. Or celle-ci n'a de sens qu'en fonction de l'usine dont elle faisait étroitement partie. C'est dire à quel point les reconversions constituent des enjeux importants et à quel point la société civile est démunie.

Autre exemple, celui de la ville de Norköpping (fig. 8), au sud de Stockholm : grande ville du textile, autrefois, désindustrialisée dans le dernier tiers du XXe siècle, elle a été profondément modifiée par la reconversion. Des immeubles d'habitation, des bureaux, une université, un musée ont été installés sur les anciens lieux industriels, suivant une recette maintenant bien connue. Le coup d'œil est magnifique. Mais un échange avec les habitants donne un tout autre son de cloche. On reproche aux architectes d'avoir joué la carte de l'esthétique contre celle de l'authenticité. D'avoir mis une distance entre ce patrimoine qui leur était si familier et ce qu'il est devenu. Ce reproche pourrait être étendu à bien des lieux qui ont fait l'objet de rénovations, depuis les fronts de mer anciens jusqu'aux centres-villes dédiés au tourisme. La « disneylandisation » guette ces endroits et surtout la banalisation, car on retrouve partout les mêmes restaurants, les mêmes boutiques, les mêmes artisans.

Figure 8 : Le centre de Norköpping

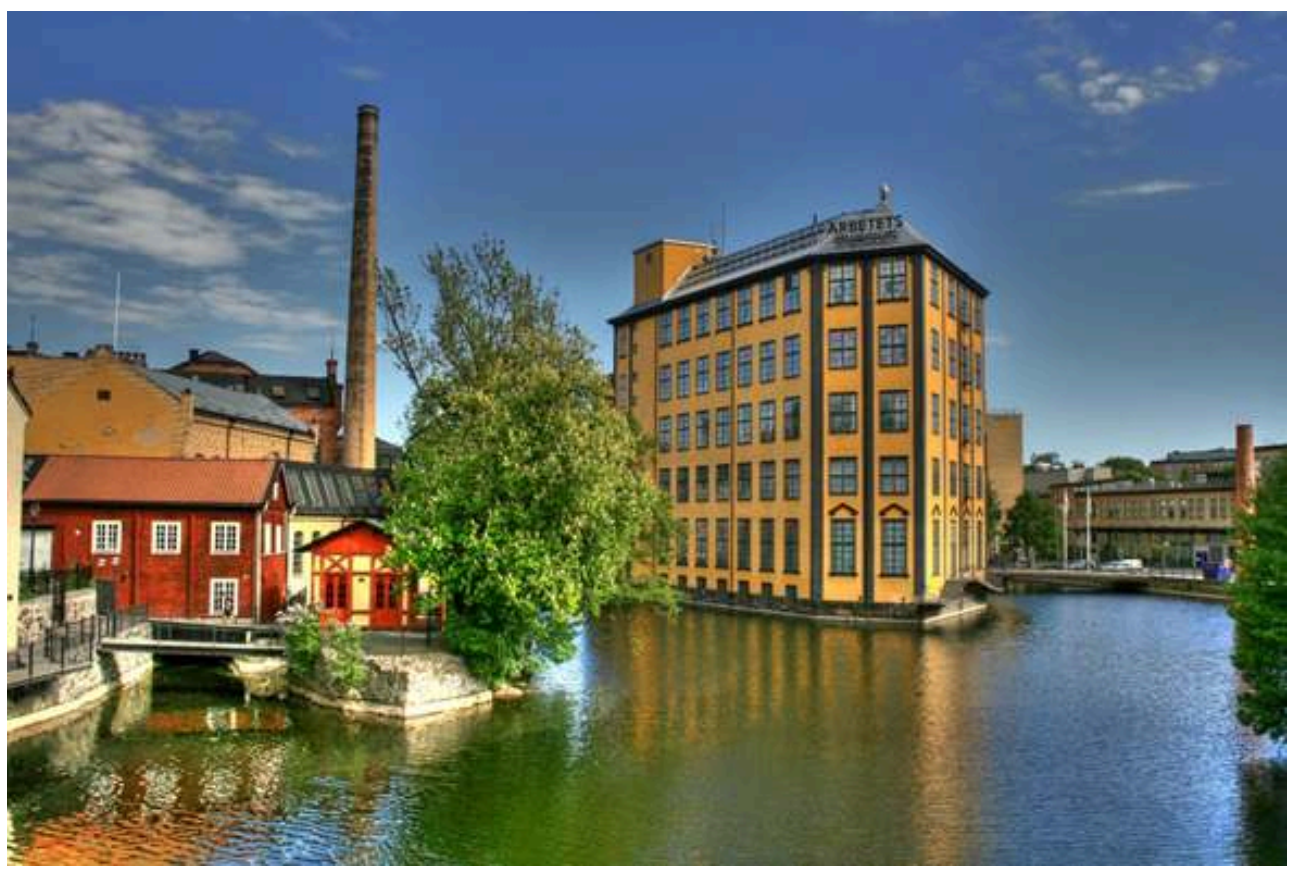

Cliché APIC

59 Le débat public a cependant son incidence sur l'évolution des approches de la part des institutions. En 2000, l'UNESCO avait ordonné une enquête approfondie sur la place du patrimoine industriel dans les nominations sur la liste du patrimoine mondial. En 2012, l'inscription du Bassin Minier du Nord-Pas de Calais a ouvert la liste à d'autres 
ensembles patrimoniaux ${ }^{7}$. En France, une législation qui donne beaucoup d'amplitude aux actions de terrain a succédé au dispositif des anciennes " villes d'art et d'histoire " et permet désormais la prendre en compte, la protection et la mise en valeur du patrimoine industriel. A partir des offices de tourisme, une documentation très complète permet au visiteur de comprendre un quartier ouvrier ou un buffet de gare des années 20...On s'approche d'une nouvelle conception du patrimoine industriel qui serait celui de l'étude de la civilisation industrielle, tout comme la civilisation de la Renaissance éclaire le XVIe siècle. Ce sont beaucoup de perspectives ouvertes pour les défenseurs de l'héritage des siècles de l'industrie et pour la projection de ce patrimoine dans un futur assumé.

\section{BIBLIOGRAPHIE}

Ahnlund M. et Brunnstrom L., 1992, « The company town in Scandinavia ». In: Garner J, The company town, architecture and society in the Early Industrial Age, New York, Oxford University Press, $229 \mathrm{p}$.

Alvarez Areces M., 2015, Vivienda obrera en la ciudad industrial del siglo XX, Incuna.

Bellat F., 2015, Une ville neuve en URSS, Togliatti, Parenthèses.

Bergeron L. 2001, “les villages ouvriers comme éléments du patrimoine de l'industrie", Patrimoine de l'industrie, $\mathrm{TICCIH}$,

Bergeron L., 2001, Le Creusot: une ville industrielle, un patrimoine glorieux. Belin-Herscher, Paris.

Bliaux F.,2016, Les monuments historiques de Saint-Gobain (en collaboration avec Francis Eck), Editions gobanaises.

Boully V. 2013,. Entre liberté d'entreprendre et surveillance par l'État. Les salines de Franche-Comté dans la seconde moitié du XIXe (1840-1907), thèse inédite, sous la direction de Dominique Barjot.

Commaille L. 1999, Les cités ouvrières de Lorraine, 1850-1940, thèse d'État.

Csaba O. 1998, A Diósgyör-vasgyári kolónia, Milkoc.

Daumalin X, Lambert O. et Mioche P., 2012, Une aventure industrielle en Camargue, Histoire de l'établissement Solvay de Salin de Giraud (1895 à nos jours), REF2 Editions.

Della Monica M. 1980, La classe ouvrière sous les pharaons, Maisonneuve.

Diffre S. 1997, Villeneuvette, 1674-1954, La manufacture royale de Villeneuvette en Languedoc, Bibliothèque42.

Dorel-Ferré G. 2006, «Les utopies industrielles: la circulation des modèles entre l'Europe et l'Amérique ». In : Daumas J.C., La mémoire de l'industrie, de l'usine au patrimoine, Presses Universitaires de Franche-Comté.

Dorel-Ferré G, 2012 « Les ingrédients de la réussite, le cas d'Almadén, Castilla-La Mancha, Espagne », L'Archéologie industrielle en France, n60, juin. 
Dorel-Ferré G., 2016 « Chemin Vert ». In : Dorel-Ferré G., Villages ouvriers et villes-usines de par le monde, Editions du Laboratoire LLSETI, Université de Savoie-Mont Blanc, pp. 144-155.

Dorel-Ferré G, 2017a, « Barcelone et son arrière-pays, le dialogue interrompu », Catalonia, Centre d'études catalanes, Université Paris-Sorbonne, revue électronique : http://www.crimic.parissorbonne.fr/catalonia-20

Dorel-Ferré G. 2017b, «Las ferroviarias en Francia durante la primera mitad del siglo XX, una vivienda de vanguardia para una élite obrera». In : Ferrari M., Actas del VI congreso internacional de Historia ferroviaria, Mendoza, http://www.ticcihargentina.com.ar/

Edelblutte S., 2010, Paysages et territoires de l'industrie en Europe, héritages et renouveaux, collection Carrefours, Ellipses.

Fontana G. L., 1997, Le vie dell'industrializzazione europea, Il Mulino.

Fontana G. L., 2003 «Dar casa agli operai. Logiche d'impresa e ingegnería sociale nell'industrializzazione moderna». In : Lussana C., Dalmine, della empresa a la città. Committenza industriale e architettura, Fondazione Dalmine.

Fortier R. - dir., 1996, Villes industrielles planifiées, Boréal.

Frouard H., 2008, Du coron au HLM, Patronat et logement social (1894-1953), Presses Universitaires de Rennes.

Garner J., 1992, The company town, architecture and society in the Early Industrial Age, New York, Oxford University Press, $229 \mathrm{p}$.

Gasnier M., 2018, Le patrimoine industriel au prisme de nouveaux défis, usages économiques et enjeux environnementaux, Presses Universitaires de Franche Comté.

Gayot G. et Lassaux B., 1984, « Manufactures et usines dans une citadelle : Sedan (XVIIe-XIXe siècle). " Revue du Nord, n³20-321, p. 495-513.

Gayot G., 1998, Les draps de Sedan. 1646-1870, Paris, Éditions de l'EHESS (avec la collaboration de Terres ardennaises).

Guerrand R-H, 1967, Les origines du logement social en France, Editions ouvrières.

Guerrand R-H, et Quillot R., 1989, Cent ans d'habitat social, une utopie réaliste, Albin Michel.

Guerrand R-H, 1992, Une Europe en construction, deux siècles d'habitat social en Europe, La Découverte.

Gueslin 1994, Les hommes du pneu : les ouvriers Michelin à Clermont-Ferrand de 1940 à 1980, Les

Éditions Ouvrières.

Hamon M. et Perrin D., 1993, Au cœur du XVIIIe siècle industriel, condition ouvrière et tradition villageoise à Saint Gobain, Paris, Editions P.A.U.

Hau M. et Stoskopf N., 2005, Les dynasties mulhousiennes, Paris.

Howard E. 1998, Les cités jardins de demain, nouvelle traduction, 11G24.

Jonas S., 1985 « La ville industrielle : questions d'identité », Revue Géographique de l'Est, tome 25, n²-3, année 1985, Vieilles villes industrielles d'Europe occidentale, p. 231-240.

Jonas S., 1994, Le Mulhouse industriel, I, L'Harmattan.

Jonas S., 2002, « Les cités jardins du Mitteleuropa ». In : Dorel-Ferré G., La cité jardin, une histoire ancienne, une idée d'avenir, Cahier de l'APIC n², p. 79-98. 
Jonas S., 2004 « Le modèle germanique du logement social et sa diffusion en Europe centrale ». In : Dorel-Ferré G. Habiter l'industrie, hier, aujourd'hui, demain, Cahier de l'APIC n²4, Reims, p. 109-117.

Leboutte R. 1997, Vie et mort des bassins industriels miniers en Europe, 1750-2000, L'Harmattan.

Ledoux Cl.-N., 1804, L'Architecture considérée sous le rapport de l'art, des mœurs et de la législation, Paris.

Le Maner Y., 1995, Du coron à la cité. Un siècle d'habitat minier dans le Nord-Pas-de-Calais, 1850-1950, Centre historique minier de Lewarde.

Lotareva R., 2011, Villes-usines russes, XVIIIe-début XIXe siècles, Socrates, Ekaterinbourg (en russe).

Lussana C., 2003, Dalmine, della empresa a la città. Committenza industriale e architettura, Fondazione Dalmine.

McLaren D. 2016, « À la recherche d'une architecture fonctionnelle, les villages ouvriers de David Dale, Ecosse, 1783-1806 ». In : Dorrel-Ferré G., Villages ouvriers et villes-usines de par le monde, Editions du Laboratoire LLSETI, Université de Savoie-Mont Blanc, p. 46-55.

Morisset L., 1998, Arvida, cité industrielle, une épopée urbaine en Amérique, Editons du Septentrion.

Nagy G.,1994, La colonie de Werkele à Budapest, Budapest.

Olajos C., 1998, A Diósgyőr-vasgyári kolónia, Miskolc.

Portal R.,1950, L'Oural au XVIIIe siècle, Paris, Institut d'Etudes Slaves, 434 p.

Poull G. 1982, L'industrie textile vosgienne (1765-1981), chez l'auteur.

Rantanen K. 2010, Living industrial past, in Tampere region, Museum Centre Vapriikki.

Rignault B.,1978, Les Forges de Buffon en Bourgogne, Association pour la sauvegarde des Forges de Buffon.

Roberts H.,1998, Des habitations des classes ouvrières, Edition revue et augmentée de 1867, édition de Michael Browne.

Rubino G. 2018, Archéologie de l'industrie ou archéologie du travail ? Villes-usines et usines doubles, deux cas significatifs du patrimoine (proto) industriel italien, Actes du colloque de TICCIH-Lille région 2015.

Scachetti E., 2013, La saline d'Arc et Senans, manufacture, utopie et patrimoine (1773-2011) thèse inédite sous la direction de Jean-Claude Daumas.

Scott J., 1942 (en français, 2010), Au-delà de l'Oural, Collection Témoignages.

Söderström O. - éd., 1997, L'industriel, l'architecte et le phalanstère, L'Harmattan.

Thomson J. K. J., 1982, Clermont de Lodève, 1633-1789, Cambridge, University Press.

Valentin M., 1994, Noisiel, la chocolaterie Menier, Seine-et-Marne, Images du Patrimoine.

Watelet H., 1993, Le Grand Hornu, joyau de la révolution industrielle et du Borinage, LebeeerHossmann.

Zola E., 1993, Carnets d'enquête, Collection Terre Humaine, Plon. 


\section{NOTES}

1. Il s'agit de The International committee for conservation of industrial heritage, fondé en 1973 à Ironbridge et depuis 2000, consultant pour l'UNESCO pour les biens relevant du patrimoine industriel, proposés sur la liste du patrimoine mondial.

2. Parler de villages ouvriers a l'avantage de mettre l'accent sur une caractéristique fonctionnelle et sociale. De plus, on retrouve ce même vocabulaire chez nos voisines européens : workers villages chez les anglais, villaggi operai chez les italiens, pueblos obreros chez les espagnols, etc. Pour la ville-usine on trouverait des équivalents : città-fabriche, ciudad fàbrica, mill town.

3. Ils sont tous les trois inscrits sur la liste du patrimoine mondial. Pour consulter les dossiers :

4. Les rapports de l'Exposition Universelle de 1867 sont consultables sur la base des archives en ligne du Conservatoire National des Arts et Métiers : cnum-cnam.fr ; sur l'expérience mulhousienne, on se reportera à la riche bibliographie sur le sujet. En particulier sur le site : http://musee-hlm.fr/discover/focus/36\#/home

5. En même temps que l'Exposition Universelle de 1889 se tient le premier congrès sur l'Habitation à bon marché, point de départ des associations de HBM qui aboutirent à créer des structures de financement pour la construction de logements sociaux en particulier à Paris.

6. Le terme de "closes" concerne les courrées de Glasgow; les «conventillos" ou petits couvents désignent paradoxalement les courées en Espagne ou en pays latino-américain.

7. En 2019, la ville-usine d'Ivrea, lieu de fabrication des machines à écrire Olivetti, a été inscrite au patrimoine mondial. Les «monts métallifères" entre l'Allemagne et la Tchéquie ont été également inscrits avec tout le patrimoine afférent, sites miniers et villes minières.

\section{RÉSUMÉS}

Dans l'histoire des agglomérations d'habitations créées par l'industrie selon ses besoins de main d'œuvre, la cité-usine tient une place à part. Sa genèse est complexe et elle évolue selon la pression d'un marché plus ou moins lointain qui stimule sa production et la pérennise. Des exemples de villes-usines non planifiées marquent l'histoire mais leur nombre s'est multiplié au XIXe siècle, avec l'accroissement de la production industrielle.

Les villes-usines planifiées, elles, sont caractéristiques du XXe siècle. Nées de toutes pièces, pour une production d'envergure, elles ont tous les attributs de la ville du fait de leur éloignement des centres urbains traditionnels.

Aujourd'hui un nombre important de ces villes-usines est dépourvu de l'activité qui les ont vu naître. Se pose alors la question de la patrimonialisation, toujours aléatoire, car elle ne correspond plus à une nécessité économique. Bien au contraire, il s'agit «d'accommoder les restes ", de celles qui ont échappé à la destruction.

In the history of housing agglomerations created by industry according to its workforce needs, the factory city holds a special place. Its genesis is complex and it evolves according to the pressure of a more or less distant market that stimulates its production and sustainability. Examples of unplanned factory towns mark history, but their numbers increased in the 19th century, with the development of the industrial production. 
Planned factory cities are characteristic of the 20th century. Born from scratch, for a large scale production, they have all the attributes of the city because of their remoteness from traditional urban centers.

Today, a significant number of these factory towns are devoid of the activity that gave birth to them. The question of their heritage dimension, which is always random, arises, because it no longer corresponds to an economic necessity. On the contrary, it is a matter of "accommodating the remains", of those who have escaped destruction.

\section{INDEX}

Keywords : workers' village, factory, factory town, miners terraced houses, garden city, industrial colony

Mots-clés : village ouvrier, manufacture, ville-usine, coron, cité-jardin, colonie industrielle

\section{AUTEUR}

\section{GRACIA DOREL-FERRÉ}

Docteure en Histoire (EHESS) - Présidente de l'Association pour le patrimoine industriel de Champagne-Ardenne (APIC) - 7 résidence de la Fournière - 51300 Marolles -

gracia.dorel@gmail.com 